\title{
Thread-like strand from right atrium into right ventricle as leader of freely moving ball thrombus
}

\author{
Jouko A. Paloheimo \\ From the Department of Medicine, Maria Hospital, Helsinki, Finland
}

A 4I-year-old man was treated in hospital four times during eight months because of cardiac palpitation, dyspnoea, and anginal pains. He had an enlarged heart, atrial fibrillation, and cardiac failure. Pulmonary embolism was suspected. After the fifth admission he died with a clinical picture of severe congestive heart failure. At necropsy a long fibrous strand was seen extending from the right atrium into the right ventricle, with a ball thrombus freely moving along the strand. In the right atrium there was a small thrombus at the base of this fibrous strand. The myocardium was hypertrophied and there were short fibrous strands between the trabeculae carneae of the ventricles of the heart. Obstruction of the right atrioventricular orifice by this ball thrombus was thought to be the cause of death.

In most cases the remnants of the Eustachian and Thebesian valves in the right atrium are of no clinical importance. These remnants may be seen as wellformed valves, as networks or threads, or they may be absent (Yater, I929; Hudson, 1965). The socalled Chiari's network has widespread attachments, from the atrial wall near the upper portion of the crista terminalis to the atrial septum or the floor of the right atrium. Remnants of the left valve of the sinus venosus may form a reticulum of fibres, in rare cases freely extending into the right atrial chamber (Gould, 1953). A well-formed valve may obstruct entry into the right atrium, especially if there is thrombus formation (Bennett, 1950; Rossall and Caldwell, 1957). It may be sutured at closure of an atrial septal defect if mistaken for the posterior inferior rim of the defect (Lewis, 1955; Hickie, 1956). The network may be the site of thrombus formation and pulmonary embolism (Chiari, 1897; Jordan, 1926; Powell and Mullaney, 1960).

The remnants of valves have their attachments in the right atrium. In the case now to be reported there was a long, thread-like strand from the upper portion of the crista terminalis through the right atrium into the right ventricle, ending at the base of a papillary muscle. A ball thrombus, with a radius of $\mathrm{I} \mathrm{cm}$, was freely moveable along the strand through the tricuspid orifice into the right ventricle and back.

\section{Case report}

The patient was a $4 \mathrm{I}$-year-old labourer. At the age of 20 he had had a goitre removed for cosmetic reasons. He had been a heavy drinker but had done hard work all his life. His father, 70, has had cardiac disease since the age of 45 and is now being treated with digitalis and propranolol. His brother, 39, has myocardial disease with paroxysmal atrial fibrillation, twice reverted to sinus rhythm with a direct-current shock. The patient had felt tired and had had cardiac palpitations some months before the age of 40 . On the first admission, at the age of 40 , he had heart pains, dyspnoea, and tiredness. His pulse was fast and irregular; no murmurs were heard. The electrocardiogram confirmed atrial fibrillation, the $Q R S$ configuration was notched, and some ventricular extrasystoles were seen. $X$-rays showed a normal heart size and slight pulmonary congestion. He was given digitalis and anticoagulants. Three weeks later three DC shocks were given without restoration of sinus rhythm. On the second admission, 5 months later, he had more symptoms; $x$-rays showed an enlarged heart and pulmonary congestion. The liver was enlarged. The electrocardiogram showed ST segment depression in leads $V_{3}-6$, not fully restored to normal after 3 weeks in hospital. Pulmonary embolism was suspected and he was treated with digitalis, diuretics, and anticoagulants. At the third admission 2 months later he again had anginal pains, tachycardia, and oedema of the legs and liver enlargement. There was a very rapid atrial fibrillation, ventricular extrasystoles, cardiac enlargement (possibly a pericardial effusion), and pulmonary congestion. Digitalis toxicity was suspected. The patient improved but was readmitted for the last time only 2 weeks after his 4-week stay in hospital. He then had severe dyspnoea, diarrhoea, and vomiting. The arterial blood pressure was between 90 and $100 \mathrm{mmHg}$ systolic and the venous pressure was high. There was rapid atrial fibrillation ( 170 a minute). The liver was enlarged three 
FIG. 2 The ball thrombus moving freely along the fibrous strand. 
fingerbreadths and was tender. The clinical picture varied from very grave venous congestion and pulmonary oedema with a high pulse frequency and low arterial pressure to a slightly better situation. After ro hours in hospital asystole occurred which did not respond to resuscitative measures.

\section{Necropsy}

The heart weighed $690 \mathrm{~g}$; both ventricles and atria were hypertrophied and dilated. The trabeculae carneae of the ventricles were hypertrophic, with dense thread-like fibrous bands $0.5-1.0 \mathrm{~cm}$ in length between them. The right atrium was enlarged and the muscle somewhat hypertrophied. The foramen ovale was closed. In the upper part of the crista terminalis there was a small, tent-like thin sail. A thin thread-like strand continued from it through the tricuspid orifice into the right ventricle, to the base of a papillary muscle (Fig. I). The small tent was surrounded by a small thrombus. A large thrombus $\mathrm{I} \mathrm{cm}$ in radius was freely moveable along the strand (Fig. 2). This thrombus was of ball form and elastic, with a small hole of the same type as the thrombus in the right atrium around the small 'tent'. No other filaments or strands were detected in the right atrium. The valves and the coronary arteries of the heart were normal. There was a fixed, nonobstructing embolus in a branch of the left pulmonary artery. The microscopical examination showed that the heart muscle was more fibrous than normal but without any specific findings. The long strand was composed of fibrous tissue only, with no muscle fibres. The thrombus fixed to the tent formation in the right atrium was old and lamellated, only a part of its lower end being fresh and red. The ball-formed thrombus also was of the same structure, with some red thrombus in its upper part. The liver was fatty and showed venous congestion.

\section{Discussion}

The case history and clinical symptoms and signs, combined with the findings at necropsy, showed that this 4I-year-old man had had myocardial disease of unknown aetiology. The cardiomyopathy exhibited the peculiar finding of short fibrous bands between the trabeculae carneae of both ventricles of the heart, and especially a long strand between the right atrium and the right ventricle, with a moveable thrombus.

The commonly seen filaments and reticular for- mations in the right atrium find their explanation in the embryonic development of the heart. The long strand seen in this patient with its tent-like attachment could also be a remnant of the phase of development when the interatrial and atrioventricular walls are formed. It is impossible to say whether it has anything to do with the short fibrous bands between the trabeculae carneae of both ventricles of the heart. The patient's high alcohol intake does not seem to offer a good explanation for this patient's myocardial disease; other cases of heart disease in the family suggest a familial cardiomyopathy.

The thrombus formation with pulmonary embolism was the most probable cause for the admissions. Loosening of the ball thrombus that moved freely through the tricuspid orifice, causing paroxysmal obstruction of the orifice, seems to be the reason for the terminal clinical picture with severe right heart insufficiency.

\section{References}

Bennett, I. L. (1950). A unique case of obstruction of the inferior vena cava. Bulletin of the fohns Hopkins Hospital, 87, 290.

Chiari, H. (1897). Net formation in the right atrium of the heart. Beiträge zur pathologischen Anatomie und zur allgemeinen Pathologie, 21, I.

Gould, S. E. (1953). Pathology of the Heart. Charles C. Thomas, Springfield, Illinois.

Hickie, J. B. (1956). The valve of the inferior vena cava. British Heart fournal, 18, 320.

Hudson, R. E. B. (1965). Cardiovascular Pathology, p. 1788. Edward Arnold, London.

Jordan, W. R. (1926). Two cases of Chiari's network. Archives of Pathology, 2, 840.

Lewis, F. J. (1955). Considerations in repair of interatrial communications (discussion). Fournal of Thoracic Surgery, 29, 52.

Powell, E. D. U., and Mullaney, J. M. (1960). The Chiari network and the valve of the inferior vena cava. British Heart Fournal, 22, 579.

Rossall, R. E., and Caldwell, R. A. (1957). Obstruction of inferior vena cava by a persistent eustachian valve in a young adult. Fournal of Clinical Pathology, 10, 40.

Yater, W. M. (1929). Variations and anomalies of the venous valves of the right atrium of the human heart. Archives of Pathology, 7, 418.

Requests for reprints to Dr. Jouko A. Paloheimo, Maria Hospital, Helsinki, Finland. 